\title{
IMPACTO EN LA SALUD MENTAL DE LA(DEL) ENFERMERA(O) QUE OTORGA CUIDADOS EN SITUACIONES ESTRESANTES
}

\author{
IMPACT ON NURSES MENTAL HEALTH GIVING CARE IN \\ STRESSFUL SITUATIONS
}

\author{
Carmen Luz Muñoz Zambrano* \\ Hossn Rumie Díaz ${ }^{* *}$ \\ Gabriela TORRes GÓMEZ *** \\ Karla Villarroel JUlio ${ }^{* * * *}$
}

\begin{abstract}
RESUMEN
La(el) Enfermera(o) tiene como principal característica la Gestión del Cuidado, es decir conservar la vida asegurando la satisfacción de las necesidades y es reconocida como el único cuidador permanente en el proceso asistencial. Las afecciones de salud mental en las(los) Enfermeras(os) están apareciendo fuertemente en la actualidad, donde destaca el estrés a la salud física, mental y emocional e incluso puede llevar a la depresión y ansiedad. Objetivo: Determinar el impacto en la salud mental de Enfermeras(os) que trabajan en situaciones estresantes en centros asistenciales de mediana y alta complejidad, públicos y privados. Material y método: Estudio no experimental, transversal, descriptivo realizado a 70 Enfermeras(os) que trabajan en Unidades de Paciente Crítico y Oncológico en la ciudad de Antofagasta, Chile, a través de un instrumento autoadministrado, escala "Nursing Stress Scale". Resultados: De las 34 situaciones estresantes aplicadas, el 48.5\% de las/los Enfermeras(os) obtuvieron puntaje mayor a 34 puntos, siendo los de mayor frecuencia: observar el sufrimiento del usuario 68,2\%; realizar procedimientos que suponen experiencias dolorosas al usuario 57,6\%; tener que realizar tareas no relacionadas con la Enfermería 56,1\% e insuficiente personal para cubrir adecuadamente el trabajo de la unidad 53\%. Conclusión: El 48.5\% de las(los) Enfermeras(os) generan algún grado de estrés, ya que otorgar cuidados en situaciones estresantes provoca impacto en la salud mental, viéndose afectados principalmente los ambientes psicológico, social y finalmente el ambiente físico.
\end{abstract}

Palabras clave: Enfermería, cuidados de enfermería, salud mental, estrés psicológico.

\footnotetext{
ABSTRACT

The Nurse have as main characteristic the "Care Management", understood as preserving life ensuring satisfaction of needs and is recognized as the only permanent caregiver in the care process. The mental health conditions in the Nurses are emerging strongly today, which highlights the stress to physical, mental and emotional health and may even lead to depression and anxiety. This research aims to determine the impact on the mental health

*Enfermera, Profesora Escuela de Enfermería Sede Puerto Montt, Universidad Austral de Chile. E-mail: carmen.munoz@ uach.cl, carmenluzmz@hotmail.com.

** Enfermera, Licenciada en Enfermería, Antofagasta, Chile. E-mail: h.rumied@gmail.com

${ }^{* * *}$ Enfermera, Licenciada en Enfermería, Antofagasta, Chile. E-mail: gabriela.alejandra.torres@gmail.com

${ }^{* * * *}$ Enfermera, Licenciada en Enfermería, Antofagasta, Chile. E-mail: karla.vj.enf@gmail.com
} 
of Nurses working in stressful situations in public and private, medium and high complexity health centers; with a non-experimental, cross-sectional study conducted at 70 Nurses working in Critical and Oncology Units patient in the city of Antofagasta, Chile, through a self-administered instrument, the scale "Nursing Stress Scale". From the 34 stressors applied, $48.5 \%$ of the nurses obtained the highest score 34 points, with the highest frequency: observe the user suffering $68.2 \%$; perform procedures involving painful experiences users $57.6 \%$ ; have to perform non- nursing work $56.1 \%$ and insufficient staff to adequately cover the work of the unit $53 \%$. Conclusion: So $48.5 \%$ of the nurses generate some degree of stress as they provide care in stressful situations resulting impact on mental health, looking mainly affected the psychological and social environment, and finally the physical environment.

Key words: Nursing, nursing care, mental health, stress, psychological.

Fecha recepción: 02/08/13 Fecha aceptación: 12/11/14

\section{INTRODUCCIÓN}

$\mathrm{La}(\mathrm{el})$ Enfermera(o) tiene como principal característica la Gestión del Cuidado, es decir conservar la vida asegurando la satisfacción de las necesidades; y es reconocida como el único cuidador permanente en el proceso asistencial $(1,2)$; pero su permanencia en el cuidado del usuario la(o) somete a situaciones estresantes y altamente vulnerable de padecer afecciones físicas y psicológicas. En el año 2008, un estudio realizado a 45 Enfermeras(os) de diferentes turnos y servicios destaca que el factor más estresante es la falta de personal, además es muy sensible al dolor o sufrimiento del usuario; entonces no es extraño encontrar en la literatura que uno de los factores que llega a producir mayor estrés es el enfrentamiento a la experiencia de la muerte y su proceso (5).

$\mathrm{Al}$ referirse al trabajo propiamente tal de la(del) Enfermera(o) se puede destacar el grado de responsabilidad que ésta tiene, ya sea por el contacto social y con los usuarios, el clima organizacional, la carga de trabajo o la necesidad de mantenimiento y desarrollo de una calificación profesional, los horarios irregulares, el ambiente físico en el que se realiza el trabajo y finalmente al hablar del género femenino se puede anexar la carga de las labores del hogar, esto tiene efectos negativos en la calidad de vida de las(los) Enfermeras(os) $(4,5)$ y aqueja su salud mental provocando estrés físico, mental y emocional e incluso puede conllevar a sufrir de depresión y ansiedad; el estrés puede provenir del cansancio y de los problemas continuos, siendo los cuerpos y mentes de las(los) Enfermeras(os) los que no tienen la oportunidad de recuperarse $(4,5)$. En el estudio "Cuidarse para no morir cuidando" (6) se indica a la(el) Enfermera(o) como único cuidador permanente en el proceso asistencial y sometida a varios factores estresantes que la convierten en un personal altamente vulnerable a padecer afecciones físicas y psicológicas si no saben canalizar adecuadamente su entrega en el proceso del cuidado enfermero.

Según el CIE la profesión de Enfermería abarca los cuidados autónomos y en colaboración que se prestan a las personas de todas las edades, familias, grupos y comunidades, enfermos o sanos (7); Florence Nightingale señala que la "Enfermería es hacerse responsable de la salud de otra persona" (8); Nightingale tuvo que resolver problemas como la falta de higiene y suciedad, problemas de hipotermia, infestaciones de parásitos y otras enfermedades oportunistas; así desde los orígenes de la profesión la(el) Enferme$\mathrm{ra}(\mathrm{o})$ ha estado sometida a situaciones de 
estrés que son propias del quehacer diario, dejando evidenciado que el cuidar demanda una condición física y psicológica adecuada al momento de brindar ayuda; al pasar el tiempo el concepto de cuidar ha evolucionado, adaptándose a diferentes momentos y acontecimientos en el mundo; Henderson lo define como "... ayudar al individuo, sano o enfermo, en la relación de aquellas actividades que contribuyen a su salud o a su recuperación (o a una muerte tranquila)"; Orem: "El arte de la Enfermería es actuar por la persona incapacitada, ayudarla a actuar por sí misma y/o ayudarla a aprender a actuar por sí misma" (8); ahora existe un reconocimiento legal, tanto en su rol propio de gestora de los cuidados como en el de colaboración médica, por lo que debe estar preparada(o) en los diversos aspectos que involucra la gestión de los cuidados, para responder con una atención de calidad ante los usuarios que demandan salud y sobre todo ante la sociedad que les ha otorgado tal responsabilidad (8$10)$, ya que el intercambio de sentimientos, pensamientos, acciones y experiencias entre la(el) Enfermera(o) y el usuario ejercen una influencia recíproca para lograr una intervención terapéutica de calidad (11).

Por otra parte, el estrés está presente en las Enfermeras(os) que trabajan en Unidades de Terapia Intensiva, ya que están en contacto permanente con el sufrimiento, el dolor, el desespero, la irritabilidad y otras reacciones que pueden surgir en los usuarios debido a que la situación de hospitalización sigue siendo un estado amenazante e incluso atemorizante (12-14); estas situaciones pueden acontecer en cualquier servicio clínico, pero se ven acentuadas en las Unidades de Cuidados Intensivos (UCI), ya que la muerte y el dolor son característicos aquí y la(el) Enfermera(o) se ve enfrentada(o) a cambios cada vez más rápidos y debe estar constantemente a la vanguardia con nuevos avances científicos y técnicos (15-21); por ello presentan algún grado de estrés, encontrándose los niveles más altos en situaciones como el conflicto con los superiores, la sobrecarga laboral y el contacto con el dolor y la muerte, como lo muestra el estudio realizado en Colombia por Zambrano (15).

Entendiendo que la salud mental es un estado de bienestar por medio del cual los individuos reconocen sus habilidades y son capaces de hacer frente al estrés normal de la vida (16), el estrés podría ser cualquier cosa que exija una respuesta no específica del cuerpo frente a cualquier demanda (17). Actualmente el estrés es un tema común en investigaciones debido a las condiciones ambientales, sociales, personales y económicas enfrentadas cotidianamente por todas las personas (18); el trabajo de la(el) Enfermera(o) se encuadra en el contexto de trabajo de riesgo, puesto que pasa la mayor parte del día sometida(o) a riesgos ocupacionales, considerados por gran parte de ellas(os) como "parte de su trabajo", afectando el autocuidado; pero se pueden realizar diferentes estrategias para prevenir y fomentar un adecuado autocuidado en las(los) Enfermeras(os) con el propósito de fortalecer o restablecer la salud y prevenir la enfermedad (19-22) como aplicar un control periódico de la evaluación y gestión del riesgo, ya que diferentes autores coinciden al decir que los profesionales de la salud en general son los que presentan mayor prevalencia de alteración en su salud mental.

Así la presente investigación pretende determinar el impacto en la salud mental de las(os) Enfermeras(os) que trabajan en situaciones estresantes en centros asistenciales de mediana y alta complejidad, públicos y privados de la ciudad de Antofagasta, durante el año 2012. Como variables se planteó: 1. Presencia de situaciones estresantes (frecuencia en que se presentan estresores laborales en el ambiente de la Enfermería hospitalaria). 2. Edad (tiempo que ha vivido una persona desde su nacimiento). 3. Estado Civil (condición particular que caracteriza a una persona en relación a sus vínculos personales con individuos de otro sexo o de su mismo sexo). 4. Género (condición orgánica masculina o 
femenina de animales o de las plantas). 5 . Antigüedad en el servicio que labora (tiempo transcurrido desde que la Enfermera comienza a trabajar en el servicio) 6. Servicio en el que labora (organización y personal destinados a cuidar intereses o satisfacer necesidades del público) y 7 . Turno en el que labora (orden según el cual se suceden varias personas en el desempeño de cualquier actividad o función).

\section{MATERIAL Y MÉTODO}

Esta investigación se fundamenta en una metodología no experimental de tipo transversal, descriptivo (23); el universo estuvo dado por 79 Enfermeras(os) que trabajaban, en la ciudad de Antofagasta, en cuatro centros asistenciales de nivel terciario públicos y privados y que cuentan con Unidades de Paciente Crítico (UPC), y un centro de nivel secundario que otorga atención a usuarios oncológicos; áreas consideradas como generadoras de estrés (24), la muestra se constituyó con 70 Enfermeras(os) que trabajan en UPC y Oncología, elegidos del universo por un muestreo no probabilístico, intencionado; utilizando un nivel de confianza de 95\% con un error de 5\%. Los criterios de inclusión fueron: 1. Enfermeras(os) que trabajen en UPC y Oncología de centros públicos y privados y 2. Enfermeras(os) que tengan un año de ejercicio profesional en el servicio clínico; estos criterios fueron elegidos debido a que el instrumento que se aplica requiere un mínimo de seis meses de ejercicio en el área de trabajo. Con el objeto establecido de un mínimo de tiempo esperable según la literatura en la que se produce la situación estresante, se les solicitó su colaboración, asegurándoles la confidencialidad en el tratamiento de la información. Para ello se les entregó un Consentimiento informado por escrito, el cuál fue aprobado y firmado, con copia para el sujeto de estudio y el investigador a fin de contar con un respaldo legal.

El instrumento de recolección de datos fue la Escala de Estrés en Enfermería de Gray-Toft y Anderson, traducida al español y validada por López ${ }^{1}$, que consta de 34 ítemes que describen distintas situaciones potencialmente causantes de estrés en el trabajo desempeñado por Enfermeras(os) a nivel hospitalario; esta escala fue aplicada preliminarmente, como piloto, en 5 Enfermeras de otros servicios clínicos de mediana y baja complejidad del Hospital de Antofagasta; los ítemes han sido agrupados en tres ambientes: físico, social y psicológico, en cada ítem las posibles respuestas son: nunca ( 0 ), a veces (1) frecuentemente (2) y muy frecuentemente (3). Sumando las puntuaciones obtenidas en cada uno de ellos se obtiene un puntaje global cuyo rango se encuentra entre 0 y 102 , de forma que a mayor puntuación mayor nivel de estresores. Además, cuenta con los antecedentes generales (edad, estado civil, sexo) y laborales (servicio en el que labora, antigüedad en éste y turno en que trabaja) del encuestado.

El estudio consideró los requisitos éticos propuestos por Ezequiel Emanuel (25) para el trabajo de evaluación de protocolos de investigación en seres humanos, desprendida de las normas del Consejo Internacional de la Organización Mundial de la Salud (CIOMS), además se contó con la autorización, por escrito del director de cada una de las instituciones involucradas y el Consentimiento informado de cada Enfermera(o) que participó de la investigación. El análisis estadístico de los datos se realiza en el software SPSS 20 en español.

${ }^{1}$ López JA. Validación de la Escala de Estrés de Enfermería (NSS), de Gray-Toft y Anderson [Memoria de Licenciatura]. [La Laguna]: Universidad de La Laguna; 2002. 122 p. 


\section{RESULTADOS}

Sobre el análisis de los aspectos generales que mide el instrumento aplicado, se obtiene que el total de la muestra, 70 Enfermeras(os), es representada por el género femenino, el cual en su mayoría corresponde al grupo etáreo adulto joven, de estado civil soltera(o), con poca antigüedad en el servicio y realizando $4^{\circ}$ turno, pertenecientes al área pública de la red asistencial de salud y en su mayoría otorgando cuidados a usuarios adultos que se encuentran en situación de riesgo vital (Tabla 1).

Tabla 1. Distribución de frecuencia características de las enfermeras.

\begin{tabular}{|c|c|c|}
\hline & Frecuencia & Porcentaje \\
\hline \multicolumn{3}{|l|}{ Género } \\
\hline Masculino & 8 & 11,4 \\
\hline Femenino & 62 & 88,6 \\
\hline Total & 70 & 100 \\
\hline \multicolumn{3}{|l|}{ Edad } \\
\hline Adulto joven & 32 & 45,7 \\
\hline Adulto medio & 21 & 30 \\
\hline Adulto maduro & 15 & 21,4 \\
\hline Adulto mayor & 2 & 2,9 \\
\hline Total & 70 & 100 \\
\hline \multicolumn{3}{|l|}{ Estado Civil } \\
\hline Soltera/o & 34 & 48,6 \\
\hline Con compromiso & 9 & 12,9 \\
\hline Casada/o & 22 & 31,4 \\
\hline Separada/o & 4 & 5,7 \\
\hline Viuda/o & 1 & 1,4 \\
\hline Total & 70 & 100 \\
\hline \multicolumn{3}{|l|}{ Antigüedad en el servicio } \\
\hline 1 a 5 años & 40 & 57,1 \\
\hline 6 a 10 años & 13 & 18,6 \\
\hline 11 a 15 años & 6 & 8,6 \\
\hline > 15 años & 11 & 15,7 \\
\hline Total & 70 & 100 \\
\hline \multicolumn{3}{|l|}{ Turno en el que trabaja } \\
\hline Administrativo & 16 & 22,9 \\
\hline Cuarto turno & 54 & 77,1 \\
\hline Total & 70 & 100 \\
\hline \multicolumn{3}{|l|}{ Tipo de usuario } \\
\hline Adulto & 51 & 72,9 \\
\hline Pediátrico & 19 & 27,1 \\
\hline Total & 70 & 100 \\
\hline \multicolumn{3}{|l|}{ Servicio en el que labora } \\
\hline Unidad de Paciente Crítico & 34 & 48,6 \\
\hline Unidad de Tratamiento Intermedio & 16 & 22,9 \\
\hline Servicio de Atención Intermedia Quirúrgica & 6 & 8,6 \\
\hline Unidad de Atención Progresiva de pacientes & 4 & 5,7 \\
\hline Cuidados Paliativos & 4 & 5,7 \\
\hline Quimioterapia Ambulatoria & 4 & 5,7 \\
\hline Radioterapia Ambulatoria & 2 & 2,9 \\
\hline Total & 70 & 100 \\
\hline
\end{tabular}


De un total de 34 situaciones estresantes, el $48.5 \%$ de las Enfermeras presentan agentes estresores que se ven relacionados con los diferentes ambientes que evalúa el instrumento (Tabla 2); los estresores con mayor frecuencia de aparición fueron: Observar el sufrimiento de un(a) paciente, correspondiente al ambiente psicológico. Realizar procedimien- tos que suponen experiencias dolorosas para los pacientes, correspondiente al ambiente psicológico. Tener que realizar muchas tareas no relacionadas con la Enfermería como tareas administrativas, correspondiente al ambiente social e Insuficiente personal para cubrir adecuadamente el trabajo de la unidad, correspondiente al ambiente físico (Tabla 3).

Tabla 2. Distribución de frecuencia de aparición de puntajes.

\begin{tabular}{lcc}
\hline & Frecuencia & Porcentaje \\
\hline Mayor a 34 puntos & 34 & 48,5 \\
Menor o igual a 34 puntos & 36 & 51,4 \\
\hline
\end{tabular}

Tabla 3. Distribución de frecuencia de agentes estresores.

\begin{tabular}{|c|c|c|c|c|c|c|c|c|c|c|}
\hline & \multicolumn{2}{|c|}{ Nunca } & \multicolumn{2}{|c|}{ Ocasionalmente } & \multicolumn{2}{|c|}{ Frecuentemente } & \multicolumn{2}{|c|}{$\begin{array}{c}\text { Muy } \\
\text { Frecuentemente }\end{array}$} & \multicolumn{2}{|c|}{ Total } \\
\hline & $\mathrm{f}$ & $\%$ & $f$ & $\%$ & $\mathrm{f}$ & $\%$ & $\mathrm{f}$ & $\%$ & $\mathrm{f}$ & $\%$ \\
\hline $\begin{array}{l}\text { Observar el sufrimiento de un } \\
\text { paciente }\end{array}$ & 3 & 4,3 & 22 & 31,4 & 28 & 40 & 17 & 24,3 & 70 & 100 \\
\hline $\begin{array}{l}\text { Realizar procedimientos que } \\
\text { suponen experiencias dolorosas } \\
\text { para los pacientes }\end{array}$ & 3 & 4,3 & 29 & 41,4 & 17 & 24,3 & 21 & 30 & 70 & 100 \\
\hline $\begin{array}{l}\text { Realizar muchas tareas no } \\
\text { relacionadas con la enfermería }\end{array}$ & 13 & 18,6 & 20 & 28,6 & 27 & 38,6 & 10 & 14,4 & 70 & 100 \\
\hline $\begin{array}{l}\text { Insuficiente personal para cubrir } \\
\text { el trabajo de la Unidad }\end{array}$ & 6 & 8,6 & 29 & 41,4 & 21 & 30 & 14 & 20 & 70 & 100 \\
\hline $\begin{array}{l}\text { No conocer lo que puede } \\
\text { comunicar a un paciente o } \\
\text { familia sobre su condición } \\
\text { médica o tratamiento }\end{array}$ & 39 & 55,7 & 24 & 34,3 & 5 & 7,1 & 2 & 2,9 & 70 & 100 \\
\hline $\begin{array}{l}\text { Dificultad al trabajar con un } \\
\text { Enfermero(a) de su propia } \\
\text { Unidad }\end{array}$ & 37 & 52,9 & 19 & 27,1 & 9 & 12,9 & 5 & 7,1 & 70 & 100 \\
\hline $\begin{array}{l}\text { Falta de oportunidad de expresar } \\
\text { a otras personas de mi Unidad } \\
\text { mis sentimientos negativos hacia } \\
\text { los pacientes }\end{array}$ & 34 & 48,6 & 27 & 38,6 & 6 & 8,6 & 3 & 4,3 & 70 & 100 \\
\hline $\begin{array}{l}\text { Falta de oportunidad de } \\
\text { compartir experiencias y } \\
\text { sentimientos con otras personas } \\
\text { de mi Unidad }\end{array}$ & 34 & 48,6 & 25 & 35,7 & 7 & 10 & 4 & 5,7 & 70 & 100 \\
\hline
\end{tabular}


Por otra parte las situaciones con menor frecuencia de aparición que generan estrés fueron: No conocer qué es lo que se puede comunicar a un paciente o familia sobre su condición médica o tratamiento, correspondiente al ambiente psicológico. Dificultad al trabajar con un(a) Enfermero(a) de su propia unidad, correspondiente al ambiente social. Falta de oportunidades para expresar a otras personas de mi unidad mis sentimientos negativos hacia los pacientes, correspondiente al ambiente psicológico y Falta de oportunidad de compartir experiencias y sentimientos con otras personas de su unidad, correspondiente al ambiente psicológico (Tabla 3).

\section{DISCUSIÓN Y CONCLUSIÓN}

La Enfermería es una profesión estresante ya que desde sus orígenes se ha visto sometida a situaciones de estrés que son propias del quehacer diario (7-10), y que con la motivación de preservar la vida y evitar el sufrimiento de aquellos que la necesiten, puede muchas veces dejar de lado su propio autocuidado para brindar y reforzar herramientas en sus usuarios. Al comienzo de este estudio se menciona la gran responsabilidad que presenta para la(el) Enfermera(o) al velar por la salud de los usuarios, el hecho de estar en contacto con el sufrimiento, el dolor, el desespero y la irritabilidad; dichas condiciones se manifiestan en los resultados ya que las situaciones que generan mayor estrés tienen relación con observar el sufrimiento de un(a) usuario(as) y realizar procedimientos que suponen experiencias dolorosas, quedando de manifiesto que el ambiente que se ve más afectado es el psicológico. Por consiguiente se presentan con mayor frecuencia las situaciones potencialmente estresantes que tienen relación con el ambiente psicológico, ya que observar el sufrimiento o el dolor en el paciente produce un alto porcentaje de estrés en los(las) Enfermeros(as), y este tipo de relaciones se consti- tuyen en eventos de intensa carga emocional para el profesional, la cual es necesario controlar cuidadosamente para que la situación pueda ser resuelta en forma eficiente. En muchos casos, estas situaciones tienen un desenlace negativo o fatal que es acompañado por sentimientos de frustración, temor, rabia o desesperación por parte del profesional, por lo que este tipo de situaciones son definidas como experiencias que ocasionan estrés mal adaptativo (26); además, el ambiente social y por último el ambiente físico resultan ser factores menos conflictivos, pero estresantes en su quehacer diario.

Esto se refuerza con los resultados del estudio realizado en Colombia (15), donde el personal de Enfermería se estresa más por conflictos con sus superiores, seguido de sobrecarga laboral, sin embargo el contacto con el dolor y la muerte de los usuarios también se encuentra entre los factores estresantes.

Al situarnos en Chile y comparar lo detectado en la ciudad de Antofagasta con un estudio realizado en Santiago, el cual describe que el personal de la salud es altamente vulnerable a padecer afecciones en su salud mental (21), destaca la relación que se establece con este estudio debido a que en promedio un $34.8 \%$ de los encuestados considera que presenta las situaciones estresantes ocasionalmente, lo que indica que se ve amenazada su salud mental por el solo hecho de presentar algún agente estresor, dicha aseveración se fundamenta aún más según lo establecido por el húngaro Selye (17), que establece que el mantenerse constantemente expuesto a estos agentes estresores puede generar una resistencia a los mismos, provocando a futuro un agotamiento y deterioro del organismo, por lo que podría verse afectada la salud mental y el autocuidado de la(del) Enfermera(o) $(18,19)$.

Por lo tanto las Enfermeras(os) que trabajan en Unidades críticas y bajo situaciones potencialmente estresantes presentan impacto en su salud mental básicamente en lo relacionado con el ámbito psicológico, por lo 
que necesitan emplear habilidades psicológicas y estrategias de afrontamiento efectivas para lograr una atención de calidad y un autocuidado también efectivo; además, se logró determinar los factores sociales y físicos que se ven alterados frente al estrés permanente al que están sometidas(os); punto relevante de mencionar es la dificultad para aplicar el instrumento, atribuyendo la falta de tiempo para responder a éste, esto reafirma lo detectado durante el análisis de los resultados, en específico de las situaciones que tienen que ver con la sobrecarga laboral por falta de tiempo y personal insuficiente para cubrir las necesidades propias exigidas por los servicios.

Resulta un gran desafío poder establecer factores psicológicos y sociales que pueden ser determinantes al momento de enfrentar efectivamente una situación estresante para que ésta no se vuelva dañina para el organismo del profesional, y que como gestoras(es) del cuidado tienen el rol protagónico también en el autocuidado, se espera que sean capaces de realizar vida sana (dormir lo suficiente, guardar una dieta equilibrada y hacer ejercicio regularmente), tener conciencia y reconocer las señales de estrés en sí mismos, identificar pensamientos, sentimientos y comportamientos cuando están bajo estrés; aún más, aprender técnicas de relajación eficaces, pensar de manera positiva, establecer prioridades y fijar límites, desarrollar el sentido del humor y compartir preocupaciones y satisfacción con miembros de la familia, similarmente con los compañeros de trabajo y amigos, y finalmente limitar el tabaco y uso de medicamentos $(5,6)$. Si se logra mantener un equilibrio constante entre las características inherentes a la organización y la(el) Enfermera(o), es probable que disminuya el estrés y los factores de riesgo desencadenantes, por lo tanto mejorará su salud mental ya que disminuirá la carga biopsicosocial que enfrenta $(16,18,22)$.

\section{REFERENCIAS}

1. García C, Martínez M. Historia de la Enfermería: La evolución histórica del cuidado Enfermero. $5^{\text {a }}$ ed. Madrid: Editorial Harcourt S.A.; 2001. 214 p.

2. Zárate R. La Gestión del Cuidado de Enfermería. Index Enferm. 2004;13 (44-45): 42-46.

3. Benbunan B, Cruz F, Roa J, Villaverde C, Benbunan B. Afrontamiento del dolor y la muerte en estudiantes de Enfermería: una propuesta de intervención. Int J Clin Health Psychol. 2007; 7(1): 197-205.

4. Vallejo A., Terranova L. Estrés Postraumático y Psicoterapia de Grupo en Militares. Ter Psicol. 2009; 27(1): 101-112.

5. Flores M, Troyo R, Valle M, Vega M. Ansiedad y Estrés en la práctica del personal de Enfermería en un hospital de tercer nivel en Guadalajara. Revista electrónica de Psicología Iztacala [Internet]. mar 2010 [citado 26 abr 2012];13(1):1-17. Disponible en: http://www.revistas.unam.mx/ index.php/repi/article/view/17075/16260

6. León C. Cuidarse para no morir cuidando. Rev Cubana Enfermer. 2007; 23(1): 15-24.

7. Consejo Internacional de Enfermeras. La definición de Enfermería [Internet]. Ginebra: Consejo Internacional de Enfermeras; actualizado 23 jun 2014 [citado 02 may 2012]. Disponible en: http:// www.icn.ch/es/about-icn/icn-definition-of-nursing/

8. Alligood M, Marriney A. Modelos y Teorías en Enfermería. $7^{\mathrm{a}}$ ed. Barcelona: Editorial Elsevier; 2011. p. 385, 225-365.

9. Código Sanitario. Decreto con Fuerza de Ley $\mathrm{N}^{\circ}$ 725. [Internet] Última versión 14. Feb 2014. Libro V, Artículo 113, inciso cuarto [citado 02 may 2012]. Disponible en: http://www.leychile.cl/Navegar?idNorma $=5595 \# 207240$ 
10. Estefo S, Paravic T. Enfermería en el rol de gestora de los cuidados. Cienc. enferm. 2010; XIV (3): 33- 39.

11. Daza R, Torres A, Prieto G. Análisis crítico del cuidado de Enfermería. Index Enferm. 2005; 14(48-49): 18-22.

12. López A, Cavalheiro A, Faria D. El Estrés de los Enfermeros que actúan en una unidad de terapia intensiva. Rev Lat Am Enfermagem. 2008; 16(1): 29-35.

13. Consejo General de Psicología de España. Estrés en padres e hijos en la hospitalización infantil. [Internet]; actualizado 2006 [citado 01 jun 2012]. Disponible en: http://www.infocop.es/view_article. asp?id $=686$

14. Hospital Clínico Universidad de Chile. Unidad de paciente Crítico. [Internet]. Redclinica.cl. actualizado 2007; acceso 06 de mayo 2012 [citado 05 may 2012]. Disponible en: http://www.redclinica.cl/ HospitalClinicoWebNeo/index.aspx?channel $=6371$

15. Zambrano G. Estresores en las Unidades de Cuidado Intensivo. Aquichan. 2006; 6(1): 89-93.

16. Organización Mundial de la Salud. Departamento de salud mental y abuso de sustancias. Inversión en Salud Mental [Internet]. Ginebra: OMS; 2006 [citado 04 may 2012]. Disponible en: http:// www.who.int/mental_health/advocacy/ en/spanish_final.pdf

17. Selye H. The evolution of the stress concept. American Scientist.1974; 61: 69299.

18. Hernández E, Cerezo S, López M, Estrategias de Afrontamiento ante el estrés Laboral en Enfermeras. Rev. Enferm. Inst.
Mex. Seguro Soc. 2007; 15; 161-166.

19. Ribera D, Cartagena de la Peña E, Reig A, Romá M, Sans I, Caruana A. Estrés laboral y salud en profesionales de enfermería: estudio empírico en la provincia de Alicante. Alicante: Secretariado de Publicaciones, Universidad de Alicante; 1993. $124 \mathrm{p}$.

20. Triviño Z, Sanhueza O. Teorías y modelos relacionados con calidad de vida en cáncer y enfermería. Aquichán, 2005. 5(1): 56-63.

21. Popp M. Estudio preliminar sobre el síndrome de burnout y estrategias de afrontamiento en enfermeras de unidades de terapia intensiva (UTI). Interdisciplinaria. 2008; 25(1):5-27.

22. Órdenes N. Prevalencia de Burnout en trabajadores del Hospital Roberto del Río. Rev Chil Pediatr. 2004; 75(5): 449454.

23. Hernández R, Fernández C, Batista $P$. Metodología de la Investigación. $5^{\mathrm{a}}$ ed. Madrid: Mc Graw-Hill; 2006. p 2-23.

24. Servicio de Salud Antofagasta [Internet]. Antofagasta: Servicio de Salud Antofagasta; 2012 [citado 30 jun 2012] Disponible en: http://ssantofagasta.redsalud.gob.cl/

25. Rodríguez E. Comités de evaluación ética y científica para las investigaciones en seres humanos y las pautas CIOMS. Acta bioeth. 2004;10(1):37-48.

26. Novoa M, Nieto C, Forero C, Caycedo E, Palma M, Montealegre M, et al. Relación entre perfil psicológico, calidad de vida y estrés asistencial en personal de enfermería. Universitas Psychologica. 2005; 4(1):63-75. 